Research Paper

\title{
Impact of CD44 expression on radiation response for bladder cancer
}

\author{
Chun-Te Wu1,2, Wei-Yu Lin'2,3, Ying-Hsu Chang4, Wen-Cheng Chen 2, 5, Miao-Fen Chen 2,5凶 \\ 1. Department of Urology, Chang Gung Memorial Hospital at Keelung, Taiwan; \\ 2. Chang Gung University, College of Medicine, Taoyuan, Taiwan; \\ 3. Department of Urology, Chang Gung Memorial Hospital at Chiayi, Taiwan; \\ 4. Department of Urology, Chang Gung Memorial Hospital at Linko, Taiwan. \\ 5. Department of Radiation Oncology, Chang Gung Memorial Hospital at Chiayi, Taiwan;
}

$\square$ Corresponding author: Miao-Fen Chen, Chang-Gung Memorial Hospital, Chiayi, \#6, Chia-Pu Rd., Putz City, Chia-Yi Hsien, Taiwan, Telephone: 886- 53621000 ext 2011, Fax: 886-5- 3623002, E-mail: miaofen@adm.cgmh.org.tw

( $)$ Ivyspring International Publisher. This is an open access article distributed under the terms of the Creative Commons Attribution (CC BY-NC) license (https://creativecommons.org/licenses/by-nc/4.0/). See http://ivyspring.com/terms for full terms and conditions.

Received: 2016.11.10; Accepted: 2017.02.24; Published: 2017.04.09

\begin{abstract}
Background Identification of potential factors that can stratify tumors' response to specific therapies will aid in the selection of cancer therapy. Radioresistance is the major obstacles to positive outcomes in bladder cancer patients after definite chemotherapy. CD44, a cancer stem cell surface marker, is relevant in treatment resistance. In the present study, we examined the role of CD44 in bladder cancer.

Methods We retrospectively analyzed the clinical outcomes of 85 bladder cancer patients treated with definite chemoradiotherapy, and correlated the expressions of CD44 with IL-6 and treatment response. Furthermore, the bladder cancer cell lines HT1 197 and MB49 were selected for cellular and animal experiments to investigate the links between the CD44, IL-6 and radiation response.

Results Analyzing the clinical specimen, the staining of CD44 was significantly linked with higher clinical stage, lower complete response rates, higher loco-regional failure rate and lower survival rate with intact bladder for patients treated with definite CCRT. In addition, the frequency of CD44 immunoreactivity was significantly higher in IL-6-positive bladder cancer specimens. By cellular experiments, the expression of CD44 was stimulated by IL-6 and linked with the cancer stem cell-like property. As demonstrated through in vitro and animal experiments using immunocompromised and immunocompetent hosts, CD44+ bladder cancer cells appeared more resistant to irradiation, associated with less $\mathrm{RT}$-induced cell death.

Conclusions Our findings suggested that CD44 is important in predicting the radiation response of bladder tumor cells. If overexpressed CD44 and/or IL-6 were noted in pre-surgical specimens, radical cystectomy is more likely to be preferred.
\end{abstract}

Key words: CD44; radiation response; cancer stem cells; bladder cancer.

\section{Introduction}

Approximately $30 \%$ of bladder cancer patients present with muscle-invasive disease (MIBC) and have an unfavorable prognosis. Radical cystectomy is the gold standard for MIBC [1], with an undeniable impact on urinary and sexual function. To spare the bladder, trimodality therapy (chemotherapy and concurrent radiation therapy (CCRT) following a complete TURBT) has been investigated as a strategy with approximately 50\% long-term disease-free survival reported in appropriately selected patients $[2,3]$. On the basis of radioresistance and cancer recurrence are the major obstacles to long-term survival, this study was undertaken to determine the potential molecular markers that can increase the ability to predict which patients will response to CCRT and disease recurrence for patients with 
muscle-invasive bladder cancer.

It is well-documented that intratumoral heterogeneity exists in human bladder cancer [4]. This heterogeneity was observed histologically and functionally by biological assays defining proliferation, anchorage-dependent growth ability and responses to therapies [5-7]. Cancer stem cells (CSCs) have been reported to play a dominant role in the resistance to current therapies leading to recurrence $[8,9]$. However, the lack of a universal CSC marker as well as the ultimate lack of correlation between the marker and the patients' resistance to therapy, is the major problem with the clinical role of CSCs. Among stem cell markers, CD44 has been shown to be relevant to resistance to treatment and to lead to tumor growth in various types of cancer [10-13]. Thus, CD44 may be a candidate biomarker for predicting radiation response and clinical outcome in bladder cancer. In the present study, we examined the role of CD44+ cancer cells in the radioresistance in vitro and in vivo and correlate the level of CD44 in clinical specimens with the treatment response and clinical outcome in MIBC patients treated with CCRT.

There is mounting evidence that proinflammatory cytokines play critical roles in the pathogenesis of bladder cancer [14, 15]. We previously observed that the levels of IL-6 were positively correlated with disease progression and prognosis in bladder cancer [16]. It has been reported that the activation of IL-6/ stat3 signaling in cancer and the tumor microenvironment induces stem cell-linked aggressiveness in cancer cells by enhancing self-renewal activity and drug resistance [17, 18]. Therefore, we also investigate the link between IL-6 signaling and CD44 to explore the molecular mechanisms and lead to more rational approaches for bladder cancer.

\section{Materials and Methods}

\section{Patient characteristics}

The Institutional Review Board of our hospital approved the present study. The written consents were signed by the patients for their specimen and information to be stored in the hospital and used for research. A total of 85 patients with muscle-invasive bladder TCC ( 39 with stage T2 and 46 with stages T3 T4) who completed a course of definite chemoradiotherapy (CCRT) treatment were enrolled in the study. On completion of CCRT, patients underwent a repeat computed tomography scans (CT) and cystoscopy examination to determine the response to treatment. Patients were observed at 3-month intervals for the first 2 years and every 6 months thereafter. The end points were overall survival (OS), disease-free survival (DFS), failure pattern and response to CCRT. Disease progression was defined as documented local recurrence or distant metastases. Analyses were performed using SPSS version 17.0.

\section{Immunohistochemical (IHC) staining}

Formalin-fixed, paraffin-embedded bladder tissues collected from bladder cancer patients who underwent cystoscopy and/or TURBT at diagnosis were subjected to immunochemical analyses. For the present study, 4-mm-thick sections were prepared from the paraffin blocks. Primary mouse monoclonal antibodies for CD44 and IL-6 were obtained from Cell Signaling Technology (Danvers, MA) and Abcam (Cambridge, MA). The IHC data for the specimens were assessed using the semi-quantitative immunoreactive score (IRS). The IRS was calculated by multiplying the staining intensity (graded as follows: $0=$ no, 1 = weak, $2=$ moderate and $3=$ strong staining) and the percentage of positively stained cells $(0=$ less than $10 \%$ of stained cells, $1=11-50 \%$ of stained cells, $2=51-80 \%$ of stained cells and $3=$ more than $81 \%$ of stained cells). An IRS scoring grade of $>=$ 2 was considered positive IHC scoring.

\section{Cell culture and reagents}

HT1197, a human bladder cancer cell line, was obtained from the American Type Culture Collection (ATCC). We maintained the bladder cancer cells in DMEM supplemented with $10 \%$ foetal bovine serum. Mouse bladder carcinoma MB49 cells were kindly gifted from Dr Yi-Wen Liu [19]. The MB49 cells were maintained in RPMI 1640 supplemented with 10\% FBS. No further authentication was conducted for the two cancer cell lines.

\section{Flow cytometry}

The cells were blocked and subsequently stained with antibodies against CD44. For apoptosis assays, tumor cells were stained with antibodies against 7-AAD and annexin V 24 hours after 6Gy irradiation. For cell cycle analysis, tumor cells were fixed and then stained with PI 24 hours after 6Gy irradiation.

\section{Ectopic and orthotopic tumor models}

This study was performed in strict accordance with the recommendations in the Guide for the Care and Use of Laboratory Animals as promulgated by the Institutes of Laboratory Animal Resources, National Research Council, USA. The protocol was approved by the Committee on the Ethics of Animal Experiments of our hospital. Eight-week-old female athymic nude mice were used as the xenograft tumor implantation model. In the ectopic tumor implantation model, $1 \times 10^{7}$ HT1197 tumor cells were 
subcutaneously implanted by injection into the dorsal gluteal region. To determine the radiosensitivity in vivo, local irradiation to 15 Gy was performed when the ectopic tumors reached $0.5 \mathrm{~cm}^{3}$ or at 2 weeks after orthotopic tumor implantation. Radiosensitivity was indicated as growth delay (i.e., the time required for the tumor to recover the previous volume after irradiation). Duplicate experiments were performed for growth delay analyses. In addition, to investigate the role of CD44 expression in immunocompetent hosts [20-21], we examined the radiation response of MB49, the corresponding syngeneic TCC cell line in C57/BL6 mice, in vivo. Eight-week-old female C57 mice were used as the tumour implantation model. In the tumor implantation model, $1 \times 10^{6}$ tumor cells were subcutaneously implanted by injection into the dorsal gluteal region.

\section{Statistical analysis}

The significance of the differences between the samples was determined using Student's t-tests. The data are presented as the means \pm standard error of the mean (SEM). All experiments, comprising three replicates, were performed at least twice indepen-

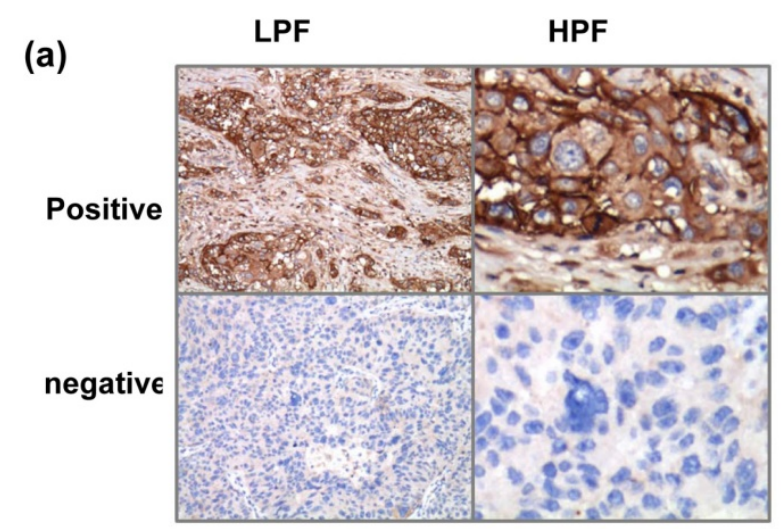

(b)

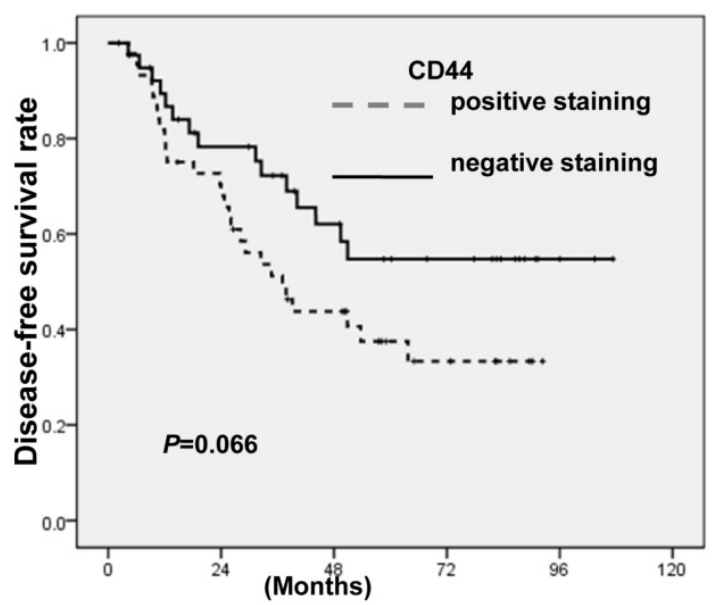

dently. A probability level of $p<0.05$ was considered statistically significant, unless otherwise stated.

\section{Results}

\section{Expression of CD44 in bladder cancer}

Figure 1a showed the representative slides of positive staining and negative staining with anti-CD44 antibody for human bladder cancer specimens at diagnosis. As listed in Table 1a, positive staining for CD44 was evident in 38\% (15/39) in T2 patients versus $65 \%(30 / 46)$ in $\mathrm{T} 3-\mathrm{T} 4, \mathrm{P}=0.013)$. The staining of CD44 was also significantly linked with higher LN metastasis, lower completed response rates and higher loco-regional failure in 85 patients treated with definite CCRT. Table $1 \mathrm{~b}$ indicated that CD44 is a significant predictor of developing disease failure after treatment on multivariate logistic regression. Furthermore, the staining of CD44 was linked with lower survival with intact bladder achieving statistical significance and diseased-free survival with borderline significance for patients treated with definite CCRT (Fig. $1 \mathrm{~b}-\mathrm{c}$ ). The findings suggested that CD44 contributes to aggressiveness and treatment resistance in bladder cancer. (c)

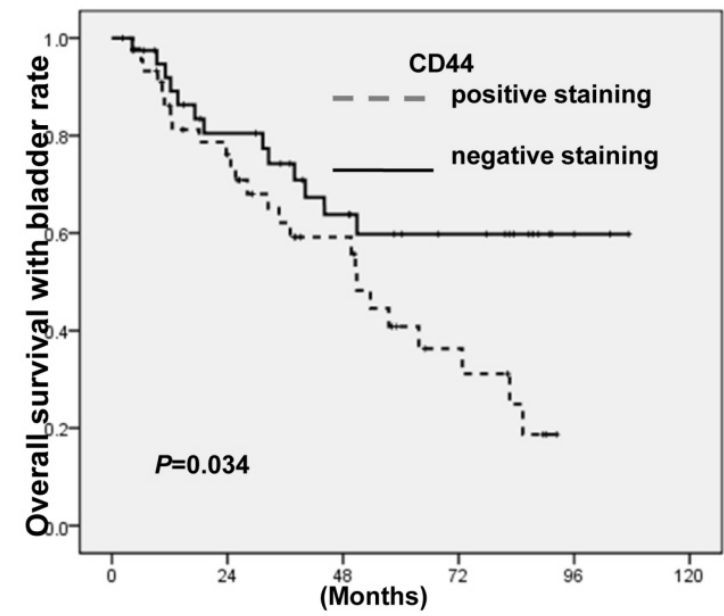

Figure 1. IHC staining for human bladder cancer specimens (a) Representative slides of IHC staining with CD44 antibodies are shown (LPF, lower power field; HPF, high power field). Survival differences according to the staining of CD44 in disease-free survival (b); and overall survival with intact bladder (c) of bladder cancer patients treated with definite CCRT. The CD44-positive group exhibited reduced survival than the CD44-negative group. 


\section{Relationship between CD44 expression and IL-6 in bladder cancer}

We previously reported that IL-6 is a significant predictor for the prognosis and treatment response of bladder cancer [16]. In the present study, we further investigate the correlation between IL- 6 and CD44. As shown in Table 1a, Table $1 \mathrm{~b}$ and Figure $2 \mathrm{a}$, positive staining for IL- 6 was evident in $51 \%$ of the 85 cancer specimens, and a significant positive correlation was found in cancer specimen that expressed CD44 and IL-6. Given the positive association between IL- 6 and CD44 expression in bladder cancer, we examined the expression of CD44 in human bladder cancer cells whose IL-6 was regulated. Flow cytometric analysis and IF data revealed that IL-6 significantly increased the level of CD44 expression at the cell surface (Figure $2 b-c)$.

\section{Expression of CD44 correlates with radiation response of bladder urothelial carcinoma}

Regarding the clinical data, the staining of CD44 was significantly linked with lower completed response rates in patients treated with definite CCRT. The findings strongly suggest that CD44 contributes to treatment resistance in bladder cancer. Therefore, the role of $\mathrm{CD} 44$ in radiation resistance and the mechanisms responsible were investigated. Colony forming assay data (Fig. 3a), Anexin-PI staining (Fig. $3 \mathrm{~b}$ ) and cell cycle analysis (Fig. 3c) demonstrated that the CD44-expressing cells was significantly resistant to irradiation associated with decreased RT-induced cell death and attenuated RT-induced G2M arrest. Furthermore, tumor xenograft with CD44-expressing cells appeared to have shorter tumor growth delay after irradiation compared to CD44 (-) tumors (Fig. $3 \mathrm{~d})$. To investigate the role of CD44 in bladder cancer in immunocompetent hosts, we examined the role of CD44 in MB49, the corresponding syngeneic TCC cell line in C57/BL6 mice, in vitro and in vivo. As shown in Figure $4 \mathrm{a}$, a higher level of CD44 was noted in IL-6 stimulated cells and sphere-forming cells than parental cells. The data from the MB49 murine ectopic tumor model (Fig. 4b) confirmed that the CD44-expressing tumor appeared more radioresistant due to shorter tumor growth delay after irradiation. The ability to carry out DNA repair is the major determinant of radiosensitivity. Attenuated RT-induced $\mathrm{p}-\mathrm{H} 2 \mathrm{AX}$, an indicator of DNA damage, was noted in CD44-expressing bladder cancer cells, compared to CD44- tumor cells, in bladder cancer cells. (a)

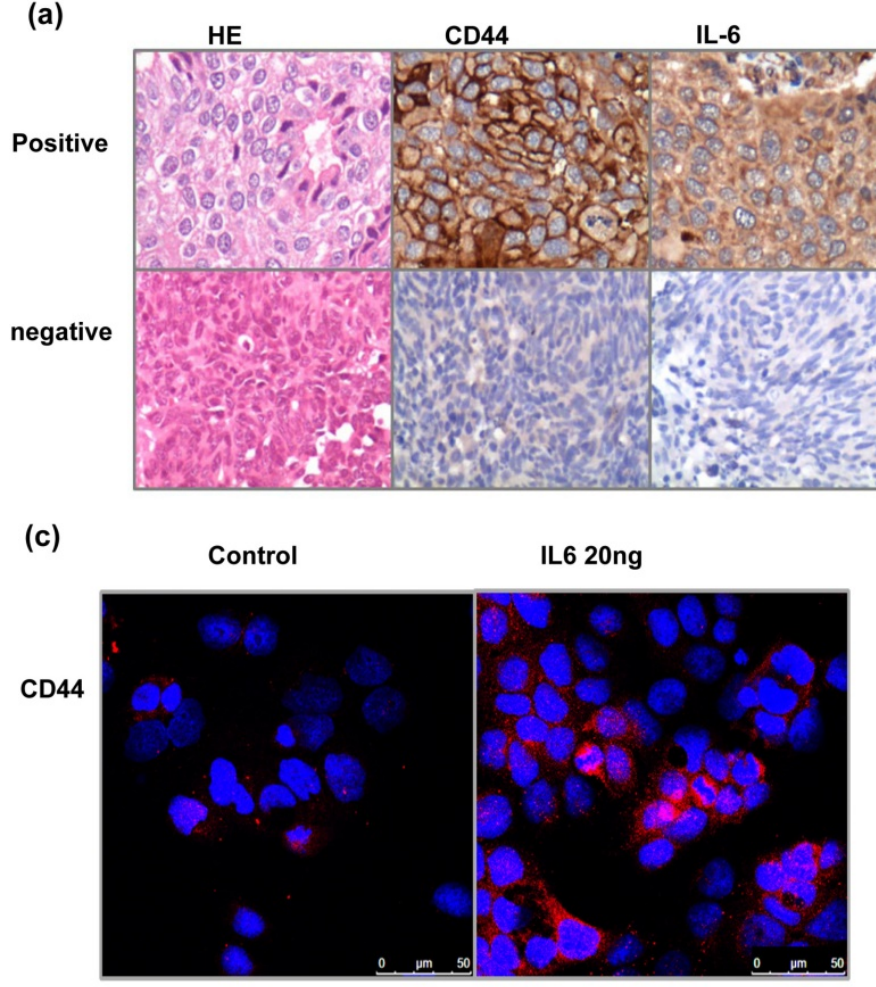

(b)
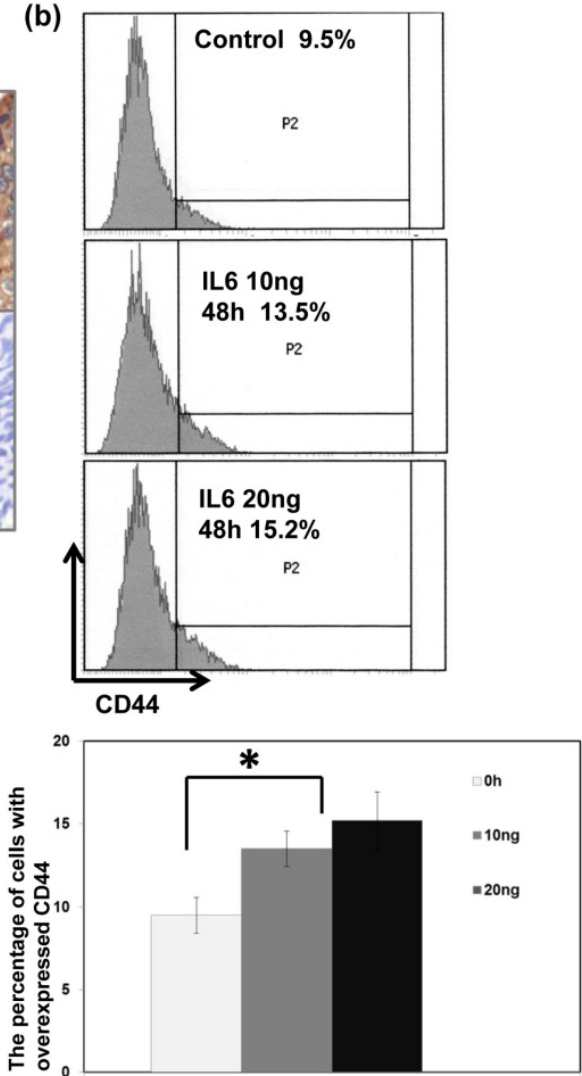

Figure 2. Correlation between CD44 and IL-6 levels in human bladder cancer cells Representative images of positive IL-6 and CD44 staining on slides from a selected tumor specimen, and representative negative staining for IL-6 and CD44 on slides from another tumor specimen, are shown. The levels of CD44 were evaluated by (b) FACS with CD44 antibody; and (c) IF staining for human bladder cancer cells 48h after IL-6 stimulation in vitro. Representative slides are shown. 


\section{Discussion}

Despite continuous improvements in cancer management, loco-regional recurrence or metastatic spread still occurs in a high proportion of patients after RT or combined treatments [22]. Understanding the molecular mechanisms that underlie the development of treatment resistance in bladder cancer is pivotal to select therapy options, including radical cystectomy and radiotherapy therapy. CSC has been reported to play a potential role in the relapse and resistance that occur in many tumors [23]. Increasing evidence indicates that CSCs contribute to radioresistance which could result in radiation treatment failure [24]. CD44, a surface adhesion molecule, has been proposed as stem cell marker. CD44 has emerged as an important gene in multiple aspects of cancer development and progression including in bladder cancer cells [4, 25-27]. The main goal of the present study was to examine the usefulness of CD44 as a novel biomarker for predicting the therapeutic outcomes of definitive CCRT in patients with MIBC, in conjunction with the expression of IL-6, which has already been reported as a significantly predictor. We correlated the level of CD44 with the clinical characteristics in bladder cancer patients treated with definite CCRT. Overexpressed CD44 was significantly associated with higher clinical tumor stage and lymph node involvement. In addition, enhanced expression of CD44 was significantly associated with a lower complete response rate after treatment and a higher disease failure rate. The data indicated a role of CD44 in predicting the treatment efficacy of CCRT for bladder sparing.

Increasing evidence suggests that the inflammatory response can be rerouted into a tumor-promoting direction, associated with poor prognosis in various types of cancer [28, 29]. IL-6 signaling has been implicated in the regulation of tumor growth and metastatic spread, and its level could be correlated with poor prognosis in different cancers [30-32]. Furthermore, it has been reported that activation of IL-6/stat3 signaling in cancer and the tumor microenvironment converted cancer cells into CSCs by enhancing self-renewal activity and drug resistance [17, 33-35]. We previously reported that IL-6 is associated with the radiation response and prognosis of bladder cancer [16]. Although only borderline significance in the DFS rate was observed between CD44+ and CD44- patients, the survival rate with intact bladder and the loco-regional control rate were indeed decreased in CD44+ patients, similar to the influence of IL- 6 expression on these outcomes.
For IHC analyses using clinical specimens, there was a positive correlation between the staining of CD44 and IL-6. Furthermore, by cellular experiments, we showed that incubation with IL-6 stimulated the expression of CD44 in bladder cancer cells, and the increase was linked with the dose of IL-6. Accordingly, we suggested that IL-6 plays a critical role in the induction of CD44+ cells in bladder cancer.

Table 1a. Baseline characteristics of patients

\begin{tabular}{|c|c|c|c|}
\hline & \multicolumn{2}{|l|}{ No. of patients } & \multirow[t]{2}{*}{$p$ value } \\
\hline & IHC-CD44 (-) & IHC-CD44 (+) & \\
\hline $\begin{array}{l}\text { Patients with } \\
\text { muscle- invasive } \\
\text { bladder cancer }\end{array}$ & 40 & 45 & \\
\hline Age & & & 0.81 \\
\hline Median & 71 & 73 & \\
\hline Range & $51-87$ & $46-92$ & \\
\hline Gender & & & 0.123 \\
\hline Male & 26 & 36 & \\
\hline Female & 14 & 9 & \\
\hline IHC-IL-6 & & & $0.0001^{*}$ \\
\hline$(-)$ & 27 & 15 & \\
\hline$(+)$ & 13 & 30 & \\
\hline Clinical stage & & & $0.013^{*}$ \\
\hline $\mathrm{T} 2$ & 24 & 15 & \\
\hline T3-T4 & 16 & 30 & \\
\hline Histologic grade & & & 0.489 \\
\hline Low & 14 & 17 & \\
\hline High & 26 & 28 & \\
\hline LN involvement & & & $0.003^{*}$ \\
\hline Negative & 37 & 30 & \\
\hline Positive & 3 & 15 & \\
\hline RT dose (cGy) & & & 0.252 \\
\hline Mean & 5833 & 5722 & \\
\hline Median & 5940 & 5940 & \\
\hline $\begin{array}{l}\text { Response to } \\
\text { definite CCRT }\end{array}$ & & & $0.031^{*}$ \\
\hline CR (+) & 34 & 29 & \\
\hline CR (-) & 6 & 16 & \\
\hline Loco-regional & & & $0.0001^{*}$ \\
\hline Control & 31 & 19 & \\
\hline Failure & 9 & 26 & \\
\hline Disease status & & & $0.007^{*}$ \\
\hline Control & 25 & 15 & \\
\hline Failure (LR+DM) & 15 & 30 & \\
\hline
\end{tabular}

Table 1b. Analysis to determine molecular markers associated with the risk of disease failure for patients treated with CCRT

\begin{tabular}{llll}
\hline Variables & $\begin{array}{l}\text { Odd } \\
\text { ratios }\end{array}$ & $\begin{array}{l}95 \% \\
\text { confidence interval }\end{array}$ & $\mathrm{p}$ \\
\hline CD44 staining & 0.294 & $0.108-0.800$ & $0.017^{*}$ \\
$\begin{array}{l}\text { Clinical stage (T2 vsT3-T4) } \\
\text { Treatment response (CR or }\end{array}$ & 2.302 & $0.798-6.643$ & 0.123 \\
$\begin{array}{l}\text { not) } \\
\text { LN involvement }\end{array}$ & 1.565 & $1.252-16.645$ & $0.021^{*}$ \\
\hline
\end{tabular}




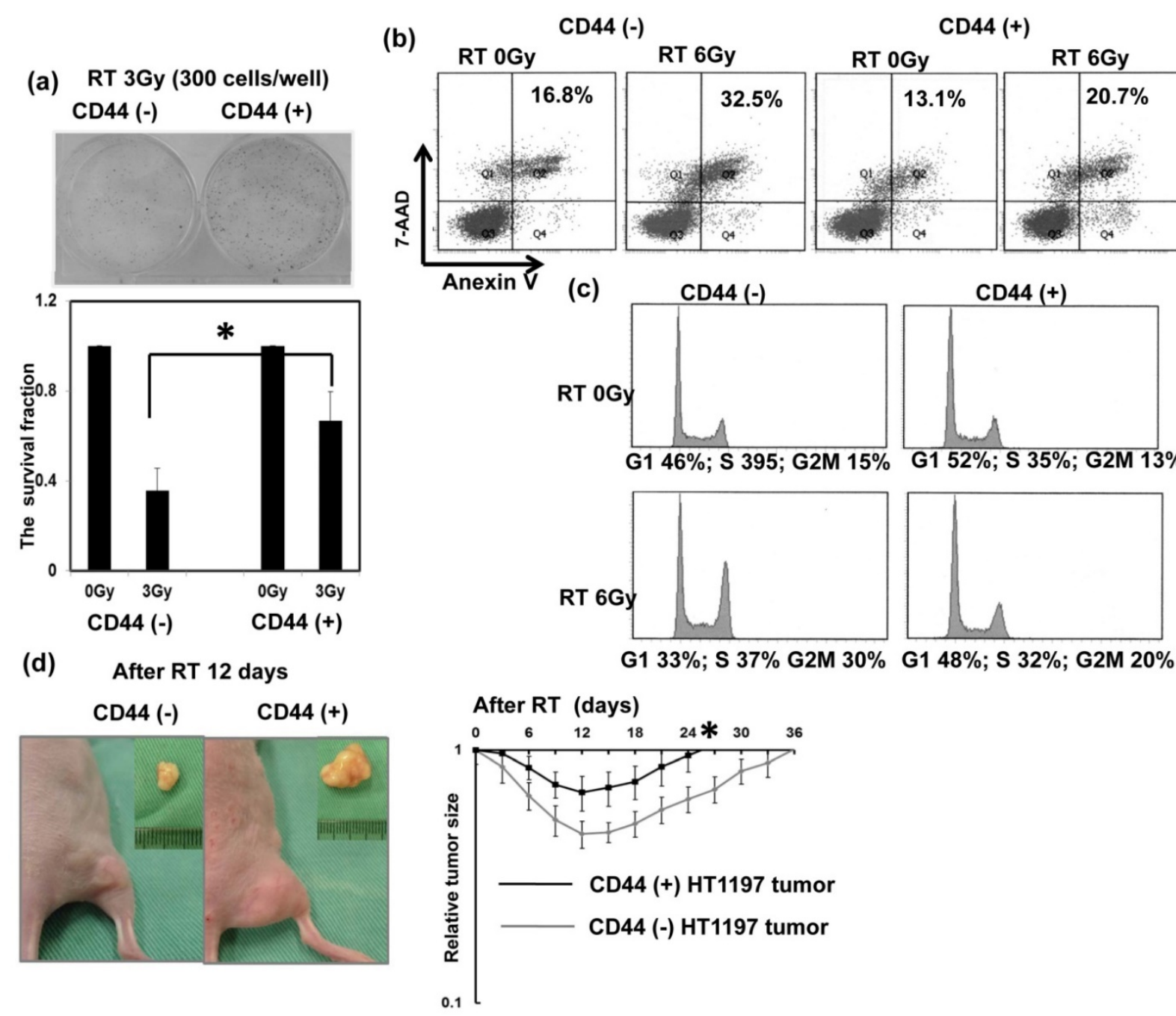

Figure 3. Role of CD44 in radiation response of human bladder cancer cells Effects of CD44 on in vitro radiosensitivity were evaluated using irradiated HT1 197 bladder cancer cells sorted by CD44 expression; (a) Clonogenic assays 7 days after 3Gy irradiation; (b) FACS with 7-ADD and Annexin V staining $48 \mathrm{~h}$ after 6Gy irradiation; (c) cell cycle analysis $48 \mathrm{~h}$ after 6Gy irradiation. Effects of CD44 on radiosensitivity in vivo were also evaluated using ectopic $\mathrm{HT} 1197$ tumor in nude mice at indicated time following 15 Gy irradiation. Points, means of 3 separate experiments; bars, SD. *, P<0.05.

Radiotherapy failure is a major problem in the treatment of malignancies. The expression of CD44 has been shown to be relevant in resistance to chemotherapy and radiation therapy, and suppression of its expression has been shown to lead to delay in tumor growth in various types of cancer [4, 11, 12, 23]. For bladder cancer, CSC-related molecular markers were reported to have potential prognostic significance for treatment response. Ferreira-Teixeira et al. [36] demonstrated that MIBC is enriched with a heterogeneous stem-like population characterized by enhanced chemoresistance. Furthermore, Koukourakis et al. [37] reported that HIF1a and LDH5 were linked with high CD44 stem cell population, and are markers of poor outcome in patients with bladder cancer treated with radiotherapy. So far, there are no reliable CSC markers in clinical practice. In the present study, we provided evidence that CD44 expression characterizes a sub-set of cancer cells with stem cell like properties and enhanced radioresistance by experiments in vitro, and in both immunocomprised and immunocompentent animal models. We also observed the predictive value of CD44 by a relatively large group of MIBC patients treated with CCRT. Regarding our clinical data, positive staining of CD44 was linked with lower complete response rate and higher disease failure rate in bladder cancer. To examine whether CD44+ cells possess the radioresistant property, the sensitivity of CD44- versus CD44+ cells to irradiation was assessed. The radiation response was evaluated using assays that take into account various types of radiation-induced cell death, specifically, in vitro clonogenic assays and in vivo tumor size measurements. The data indicated that the survival rates of CD44+ cells were higher under irradiation compared with CD44- cells. Extensive DNA damage caused by radiation that is left unrepaired can result 
in cell death or clinical radiation sensitivity [38]. We demonstrated that CD44+ cells were associated with decreased RT-induced cell death, and attenuated the expression of p-H2AX, an indicator of double-strand breaks, induced by irradiation. Furthermore, by cell cycle analysis [39], more cells were arrested in the G1 phase of the cell cycle in CD44+ cancer cells with radiation compared to CD44- HT1197 bladder cancer cells. The results validated a role for CD44+ cells in radioresistance, which might explain the failure of current therapies to eradicate cancer cells and prevent tumor recurrence.

Emerging evidence suggests that the generation of antitumor immune responses might play an important role in tumor growth and the effectiveness of radiotherapy. To address this issue, we examined the significance of CD44 expression in bladder cancer in immunocompetent hosts. The data revealed that implanted CD44+ tumors appeared more aggressive noted by murine orthotopic bladder tumor, and higher resistance to radiation by ectopic tumors with a shorter tumor growth delay after irradiation.
Therefore, the data both in the immunocompromised and immunocompetent host indicated that CD44+ cells play an important role in the treatment outcome of bladder cancer.

These findings indicate that the expression of CD44 positive linked with the development of treatment resistance in bladder cancer, and activated IL-6 signaling provides a suitable microenvironment for the induction of CD44 expression. Thus a prospective study that analyzes the expression of CD44 and IL-6 in tumor tissue may allow more accurate evaluation of the predictive ability for therapeutic outcome in bladder cancers. If CD44 and/or IL-6 expression levels could be measured in the serum or urine with the same predictive properties derived from bladder cancer tissue, the application of the former is likely to be preferred. Furthermore, novel immune-based therapies for the treatment of cancer are currently under development. Targeting CD44 directly and/or indirectly by IL-6 signaling could be a promising strategy to improve the prognosis for bladder cancer. (a)

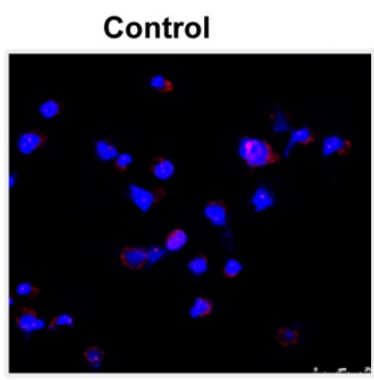

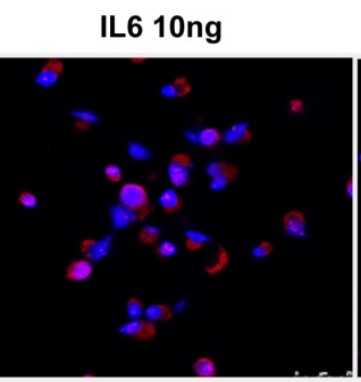

Sphere-forming

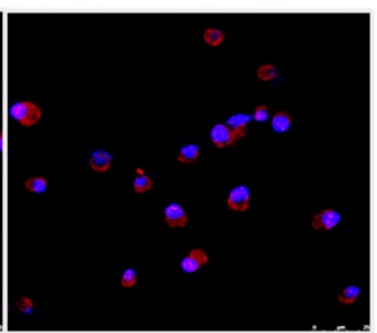

(b)

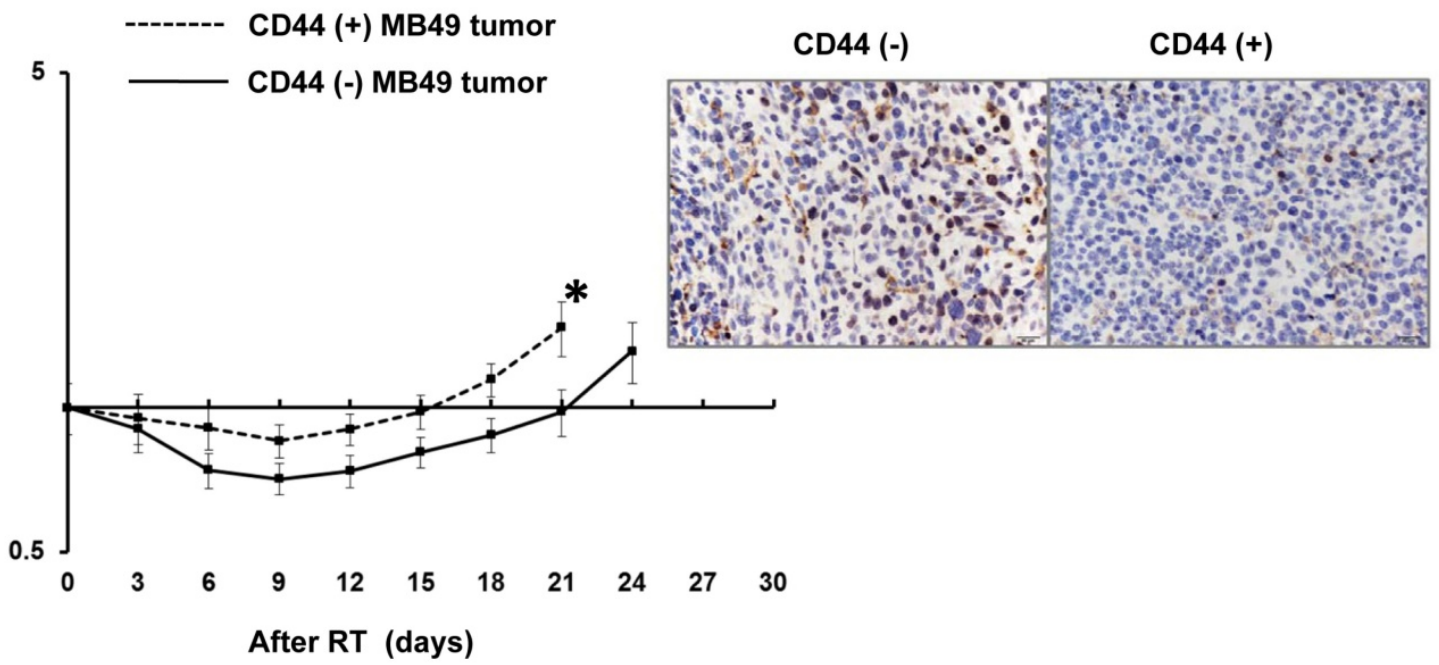

Figure 4. Expression of CD44 in murine MB49 bladder cancer cells in vitro and in vivo Levels of CD44 were evaluated by IF staining for murine MB49 bladder cancer cells $48 \mathrm{~h}$ after IL-6 stimulation and sphere-forming cells in vitro (a). Representative slides are shown. Effects of CD44 on radiosensitivity in vivo were also evaluated using ectopic MB49 tumor in immunocompetent c57 mice at indicated time following $15 \mathrm{~Gy}$ irradiation (b). IHC analysis with $\mathrm{P}-\mathrm{H} 2 \mathrm{AX}$ antibody were also evaluate $48 \mathrm{~h}$ after irradiation. 


\section{Acknowledgements}

The work was support by Chang Gung Memorial Hospital, Taiwan, grant OMRPG6C0011 and CMRPG6D0323 (to M.F. Chen) and CMRPG 2D0272 (to C.T. $\mathrm{Wu}$ ) and National Science Council, Taiwan. Grant NMRPG2E6031 (to C.T. Wu).

\section{Competing Interests}

The authors have declared that no competing interest exists.

\section{References}

1. Stein JP, Lieskovsky G, Cote R, et al. Radical cystectomy in the treatment of invasive bladder cancer: long-term results in 1,054 patients. J Clin Oncol 2001; 19: 666-675.

2. Rodel C, Weiss C, Sauer R. Trimodality treatment and selective organ preservation for bladder cancer. J Clin Oncol 2006; 24: 5536-5544.

3. Zapatero A, Martin de Vidales C, Arellano R, et al. Updated results of bladder-sparing trimodality approach for invasive bladder cancer. Urol Oncol 2010; 28: 368-374.

4. Chan KS, Volkmer JP, Weissman I. Cancer stem cells in bladder cancer: a revisited and evolving concept. Curr Opin Urol 2010; 20: 393-397.

5. Lipponen PK, Eskelinen MJ, Nordling S. Intratumoral heterogeneity of DNA indexes in transitional cell bladder cancer: relation to tumor histology. Eur Urol 1991; 20: 311-314

6. Kovnat A, Buick RN, Connolly JG, et al. Comparison of growth of human bladder cancer in tissue culture or as xenografts with clinical and pathological characteristics. Cancer Res 1984; 44: 2530-2533.

7. Anderson $\mathrm{KC}$, Simpson WG, Ballou RJ, et al. In vitro chemosensitivity of J-82 human bladder cancer cells. Urol Res 1986; 14: 141-144.

8. Yu SC, Bian XW. Enrichment of cancer stem cells based on heterogeneity of invasiveness. Stem Cell Rev 2009; 5: 66-71.

9. Frank NY, Schatton T, Frank MH. The therapeutic promise of the cancer stem cell concept. J Clin Invest 2010; 120: 41-50.

10. Hatina J, Schulz WA. Stem cells in the biology of normal urothelium and urothelial carcinoma. Neoplasma 2012; 59: 728-736.

11. Ponta $\mathrm{H}$, Sherman $\mathrm{L}$, Herrlich PA. CD44: from adhesion molecules to signalling regulators. Nat Rev Mol Cell Biol 2003; 4: 33-45.

12. Okada H, Yoshida J, Sokabe M, et al. Suppression of CD44 expression decreases migration and invasion of human glioma cells. Int J Cancer 1996; 66: 255-260

13. Koukourakis MI, Giatromanolaki A, Tsakmaki V, et al. Cancer stem cell phenotype relates to radio-chemotherapy outcome in locally advanced squamous cell head-neck cancer. Br J Cancer 2012; 106: 846-853.

14. Zhu Z, Shen $Z, X u C$. Inflammatory pathways as promising targets to increase chemotherapy response in bladder cancer. Mediators Inflamm 2012; 2012: 528690 .

15. Smyth MJ, Cretney E, Kershaw MH, et al. Cytokines in cancer immunity and immunotherapy. Immunol Rev 2004; 202: 275-293.

16. Chen MF, Lin PY, Wu CF, et al. IL-6 expression regulates tumorigenicity and correlates with prognosis in bladder cancer. PLoS One 2013; 8: e61901.

17. Korkaya H, Kim GI, Davis A, et al. Activation of an IL6 inflammatory loop mediates trastuzumab resistance in HER2+ breast cancer by expanding the cancer stem cell population. Mol Cell 2012; 47: 570-584

18. Silver DJ, Sinyuk M, Vogelbaum MA, et al. The intersection of cancer, cancer stem cells, and the immune system: therapeutic opportunities. Neuro Oncol 2016; 18: 153-159.

19. Shen $\mathrm{CH}$, Shee JJ, Wu JY, et al. Combretastatin A-4 inhibits cell growth and metastasis in bladder cancer cells and retards tumour growth in a murine orthotopic bladder tumour model. Br J Pharmacol 2010; 160: 2008-2027.

20. Wu CT, Chen WC, Chang $\mathrm{YH}$, et al. The role of PD-L1 in the radiation response and clinical outcome for bladder cancer. Sci Rep 2016; 6: 19740.

21. Wu CT, Lin WY, Chang YH, et al. DNMT1-dependent suppression of microRNA424 regulates tumor progression in human bladder cancer. Oncotarget 2015; 6: 24119-24131.

22. Koch U, Krause M, Baumann M. Cancer stem cells at the crossroads of current cancer therapy failures--radiation oncology perspective. Semin Cancer Biol 2010; 20: 116-124.

23. Gerweck LE, Wakimoto H. At the Crossroads of Cancer Stem Cells, Radiation Biology, and Radiation Oncology. Cancer Res 2016; 76: 994-998.

24. Rycaj K, Tang DG. Cancer stem cells and radioresistance. Int J Radiat Biol 2014; 90: 615-621.

25. Jeong BJ, Liang ZL, Huang SM, et al. CD44 is associated with tumor recurrence and is an independent poor prognostic factor for patients with localized clear cell renal cell carcinoma after nephrectomy. Exp Ther Med 2012; 3: 811-817.
26. Lin $\mathrm{Y}$, Zhong $\mathrm{Y}$, Guan $\mathrm{H}$, et al. CD44+/CD24- phenotype contributes to malignant relapse following surgical resection and chemotherapy in patients with invasive ductal carcinoma. J Exp Clin Cancer Res 2012; 31: 59.

27. Sugiyama M, Woodman A, Sugino T, et al. Non-invasive detection of bladder cancer by identification of abnormal CD44 proteins in exfoliated cancer cells in urine. Clin Mol Pathol 1995; 48: M142-147.

28. Vakkila J, Lotze MT. Inflammation and necrosis promote tumour growth. Nat Rev Immunol 2004; 4: 641-648.

29. Mantovani A, Allavena P, Sica A, et al. Cancer-related inflammation. Nature 2008; 454: 436-444.

30. Schafer ZT, Brugge JS. IL-6 involvement in epithelial cancers. J Clin Invest 2007; 117: 3660-3663.

31. Santer FR, Malinowska K, Culig Z, et al. Interleukin-6 trans-signalling differentially regulates proliferation, migration, adhesion and maspin expression in human prostate cancer cells. Endocr Relat Cancer 2010; 17: 241-253

32. Chen MF, Kuan FC, Yen TC, et al. IL-6-stimulated CD11b+ CD14+ HLA-DRmyeloid-derived suppressor cells, are associated with progression and poor prognosis in squamous cell carcinoma of the esophagus. Oncotarget 2014; 5: 8716-8728.

33 Boyle ST, Kochetkova M. Breast cancer stem cells and the immune system: promotion, evasion and therapy. J Mammary Gland Biol Neoplasia 2014; 19: 203-211.

34. Mitchem JB, Brennan DJ, Knolhoff BL, et al. Targeting tumor-infiltrating macrophages decreases tumor-initiating cells, relieves immunosuppression, and improves chemotherapeutic responses. Cancer Res 2013; 73: 1128-1141.

35. Yang J, Liao D, Chen C, et al. Tumor-associated macrophages regulate murine breast cancer stem cells through a novel paracrine EGFR/Stat3/Sox-2 signaling pathway. Stem Cells 2013; 31: 248-258.

36. Ferreira-Teixeira M, Parada B, Rodrigues-Santos P, et al. Functional and molecular characterization of cancer stem-like cells in bladder cancer: a potential signature for muscle-invasive tumors. Oncotarget. 2015;6:36185-201.

37. Koukourakis MI, Kakouratos C, Kalamida D, et al. Hypoxia-inducible proteins HIF1a and lactate dehydrogenase LDH5, key markers of anaerobic metabolism, relate with stem cell markers and poor post-radiotherapy outcome in bladder cancer. Int J Radiat Biol. 2016;92:353-63

38. Pouliliou S, Koukourakis MI. Gamma histone 2AX (gamma-H2AX)as a predictive tool in radiation oncology. Biomarkers 2014; 19: 167-180.

39. Chang L, Graham P, Hao J, et al. Cancer stem cells and signaling pathways in radioresistance. Oncotarget 2016; 7: 11002-11017. 